\title{
Global Dynamics of a Periodic SEIRS Model with General Incidence Rate
}

\author{
Eric Ávila-Vales, Erika Rivero-Esquivel, and Gerardo Emilio García-Almeida \\ Facultad de Matemáticas, Universidad Autónoma de Yucatán, Anillo Periférico Norte, Tablaje 13615, 97119 Mérida, YUC, Mexico
}

Correspondence should be addressed to Eric Ávila-Vales; avila@correo.uady.mx

Received 25 July 2017; Revised 3 October 2017; Accepted 10 October 2017; Published 9 November 2017

Academic Editor: Khalid Hattaf

Copyright (c) 2017 Eric Ávila-Vales et al. This is an open access article distributed under the Creative Commons Attribution License, which permits unrestricted use, distribution, and reproduction in any medium, provided the original work is properly cited.

\begin{abstract}
We consider a family of periodic SEIRS epidemic models with a fairly general incidence rate of the form $S f(I)$, and it is shown that the basic reproduction number determines the global dynamics of the models and it is a threshold parameter for persistence of disease. Numerical simulations are performed using a nonlinear incidence rate to estimate the basic reproduction number and illustrate our analytical findings.
\end{abstract}

\section{Introduction}

Epidemiological models in mathematics have been recognized as valuable tools in analyzing the dynamics of an infectious disease nowadays. They are used to describe the spread of disease and also to make control measures known to avoid its persistence, for example, via vaccination terms or treatment terms. These models consider the total population divided into compartments, given by the biological assumptions on the model and represented by functions depending on time $t$. The most common categories used are susceptible $(S)$, infected $(I)$, recovered $(R)$, exposed $(E)$, quarantined $(Q)$, and vaccinated $(V)$, and the dynamics of model is given by transmission rates from a compartment to another. We have then indicated that the models could be of type SIR, SIRS, SEIR, SEIRS, SEIVR, SEIQV, and so forth.

To ensure that the model can give a justified qualitative description of the disease, the choice of the incidence rate plays an important role. An incidence rate is defined as the number of new health related events or cases of a disease in a population exposed to the risk in a given time period. Some examples are the bilinear incidence rate, the saturated incidence rate, or a general incidence rate. The bilinear incidence rate has been repeatedly used by several authors. It is given by $\beta S I$, where $\beta$ is the transmission rate and the product SI represents the contact between infected and susceptible individuals (based on the law of mass action).
It was introduced by Kermack and McKendrick [1] in 1927, and even when it is mathematically simple to use, it faces multiple problems and challenges when it is used to describe disease propagation among gregarious animals or persons [2], because it goes to infinity when $I$ becomes larger. In order to improve the modelling process to study the dynamics of infection among a large population, Capasso and Serio [3] in 1978 introduced a saturated incidence rate by studying the Cholera epidemic spread in Bari, given by $\beta S I /(1+k I)$, where $\beta$ is the transmission rate and $k$ the saturation constant. Unlike the bilinear incidence, saturated incidence does not grow up without a limit, but it goes to a saturation limit as $I$ goes to infinity. Multiple types of saturated incidence have been used in the literature; see, for example, [2] for a list of them. To avoid the use of a single incidence function, the use of a general incidence rate that includes a family of particular functions with similar properties has become a topic of interest by several authors (see, e.g., [4-8]).

The basic reproduction (represented by $\mathscr{R}_{0}$ ) is defined as "the average number of secondary cases produced by a single infected case when it is introduced in a susceptible population" and it has an important role in the study of disease transmission. In biological terms, usually when this number is less than one, the disease is eradicated from population, but when it is greater than one, the infection persists. Mathematically, it is of interest to compute a threshold parameter with the properties of the basic reproduction number. A 
method to compute this number for certain compartmental disease models is via the next-generation matrix method developed in [9]; however, it is not useful when the model presents time periodic seasonal terms. Authors like $[10,11]$ have defined its basic reproduction number for periodic models as an average, to give some results about extinction or persistence of infection. However Bacaër and Guernaouni in [12] introduced the definition of basic reproduction number for periodic environments, and, later, Wang and Zhao [13] made a formal definition of it, via the monodromy matrix.

In the present work, we focus on a family of SEIRS epidemic models with a time periodic seasonal term, improving the model of Moneim and Greenhalgh in [14], by introducing an incidence rate with a general function taken from [4] and the references therein.

We propose the following SEIRS model:

$$
\begin{aligned}
& \frac{d S}{d t}=\mu N(1-p)-\beta(t) S f(I)-(\mu+r(t)) S+\delta R \\
& \frac{d E}{d t}=\beta(t) S f(I)-(\mu+\sigma) E \\
& \frac{d I}{d t}=\sigma E-(\mu+\gamma) I \\
& \frac{d R}{d t}=\mu N p+r(t) S+\gamma I-(\mu+\delta) R
\end{aligned}
$$

where $N=S+E+I+R$ is the total population size, with $S, E, I, R$ denoting the fractions of population that are susceptible, exposed, infected, and recovered, respectively. $\beta(t)$ is the transmission rate and it is a continuous, positive $T$-periodic function. $p(0 \leq p \leq 1)$ is the vaccination rate of all newborn children. $r(t)$ is the vaccination rate of all susceptibles in the population and it is a continuous, positive periodic function with period $L T$, where $L$ is an integer. $\mu$ is the common per capita birth and death rate. $\sigma, \gamma$, and $\delta$ are the per capita rates of leaving the latent stage, infected stage, and recovered stage, respectively. It is assumed that all parameters are positive constants.

Bai and Zhou in [5] answered some open problems stated in [14], they also showed that their condition is a threshold between persistence and extinction of the disease via the framework established in [13]. They assumed that the incidence was bilinear. In our study, the nonlinear assumptions on function $f$ are listed below (see [4]).

(A1) $f: \mathbb{R}_{+} \rightarrow \mathbb{R}_{+}$is continuously differentiable.

(A2) $f(0)=0, f^{\prime}(0)>0$ and $f(I)>0$ for all $I>0$.

(A3) $f(I)-I f^{\prime}(I) \geq 0$.

Under these assumptions, function $f(I)$ includes various types of incidence rate; in particular, when $f(I)=I$, we are on the bilinear case considered in [14]. [15]).

In addition, we assume the following extra conditions (see

(A4) $f^{\prime \prime}(0) \leq 0$.

(A5) There exists $\epsilon^{*}>0$ such that when $0<I<\epsilon^{*}, f(I) \geq$ $f(0)+I f^{\prime}(0)+(1 / 2) I^{2} f^{\prime \prime}(0)$.
This set of assumptions on the function $f$ allows for more general incidence functions than the bilinear one, like saturated incidence functions and functions of the form $\beta S I /\left(1+k I^{q}\right)$; in particular, in the case when $q>1$, they represent psychological or media effects depending on the infected population. In this last case the incidence function is nonmonotone on $I$. (A3) regulates the value of $f(I)$ comparing it with the value at $I$ of a line containing the origin of slope $f^{\prime}(I)$ (note that this line varies as $I$ increases), (A4) requires a concave $f(I)$ at the origin, and (A5) imposes the geometrical condition that in a small neighborhood of the origin $f(I)$ must lie between the tangent line of $f$ at $I$ and a concave parabola tangent to $f$ at $I$.

We consider a family of SEIRS epidemic models with periodic coefficients and general incidence rate in epidemiology. Then we show that the global dynamics of solutions is determined by the basic reproduction number $\mathscr{R}_{0}$, generalizing the results in [5]. The layout of this paper is as follows: In Section 2, we prove the existence of a disease-free periodic solution and we introduce the basic reproduction number via the theory developed in $[12,13]$. In Section 3, we adapt the arguments given in [5] to prove that the disease-free periodic solution of system (1) is globally asymptotically stable if $\mathscr{R}_{0}<$ 1 and it is persistent when $\mathscr{R}_{0}>1$. Finally, in Section 4, we give some numerical simulations of our results, making a comparison between our basic reproduction number $\mathscr{R}_{0}$ and the average reproduction number $\mathscr{R}_{0}^{T}$ used by several authors (see, e.g., $[10,11])$.

\section{The Basic Reproduction Number}

First of all, we prove nonnegativity of the solutions under nonnegative initial conditions.

Theorem 1. Let $S_{0}, E_{0}, I_{0}, R_{0} \geq 0$. The solution $(S(t), E(t), I(t)$, $R(t))$ of (1) with

$$
(S(0), E(0), I(0), R(0))=\left(S_{0}, E_{0}, I_{0}, R_{0}\right)
$$

is nonnegative in the sense that $S(t), E(t), I(t), R(t) \geq 0, \forall t>0$, and satisfies $S(t)+E(t)+I(t)+R(t)=N$, with $N$ constant.

Proof. Let $N(t)=S(t)+E(t)+I(t)+R(t)$; then, adding all equations of system (1), we can see that $d N / d t=0$, so the value of $N$ is constant. Now, set $x(t)=(S(t), E(t), I(t), R(t))$ as the solution of system (1) under initial conditions $x_{0}=$ $(S(0), E(0), I(0), R(0))=\left(S_{0}, E_{0}, I_{0}, R_{0}\right) \geq 0$. By the continuity of solutions, for all of $S(t), E(t), I(t)$ and $R(t)$ that have a positive initial value at $t=0$, we have the existence of an interval $\left(0, t_{0}\right)$ such that $S(t), E(t), I(t), R(t) \geq 0$ for $0<t<t_{0}$. We will prove that $t_{0}=\infty$.

If $S\left(t_{1}\right)=0$ for a $t_{1} \geq 0$ and other components of $x(t)$ remain nonnegative at $t=t_{1}$, then

$$
\frac{d S}{d t}\left(t_{1}\right)=\mu N(1-p)+\delta R\left(t_{1}\right) \geq 0
$$

implying that whenever the solution $x(t)$ touches the $S$-axis, the derivative of $S$ is nondecreasing and the function $S(t)$ does 
not cross to negative values. Similarly, when $E\left(t_{1}\right)=0$ for a $t_{1} \geq 0$ and other components remain nonnegative, we have

$$
\frac{d E}{d t}\left(t_{1}\right)=\beta\left(t_{1}\right) S\left(t_{1}\right) f\left(I\left(t_{1}\right)\right) \geq 0 .
$$

When $I\left(t_{1}\right)=0$ for $t_{1} \geq 0$ and other components remain nonnegative,

$$
\frac{d I}{d t}\left(t_{1}\right)=\sigma E\left(t_{1}\right) \geq 0
$$

Finally, when $R\left(t_{1}\right)=0$ for a $t_{1} \geq 0$ and other components remain nonnegative,

$$
\frac{d R}{d t}\left(t_{1}\right)=\mu N p+r\left(t_{1}\right) S\left(t_{1}\right)+\gamma I\left(t_{1}\right) \geq 0 .
$$

Therefore, whenever $x(t)$ touches any of the axes $S=0$, $E=0, I=0$, or $R=0$, it never crosses them.

In order to make the analysis of the model in a simpler way from now on, we make a reduction of dimension in system (1) making $R=N-S-E-I$, obtaining the following:

$$
\begin{aligned}
\frac{d S}{d t}= & \mu N(1-p)-\beta(t) S f(I)-(\mu+r(t)) S \\
& +\delta(N-S-E-I), \\
\frac{d E}{d t}= & \beta(t) S f(I)-(\mu+\sigma) E, \\
\frac{d I}{d t}= & \sigma E-(\mu+\gamma) I .
\end{aligned}
$$

The dynamics of system (1) is equivalent to that of (7); moreover, due to positivity of solutions, we have $S+E+I \leq N$, so we study the dynamics of system (7) in the region

$$
X=\left\{(S, E, I) \in \mathbb{R}_{+}^{3}: S+E+I \leq N\right\} .
$$

A disease-free periodic solution can be found for (7). To find it, set $E=I=0$; then, from the first equation of (7) we can obtain the following initial value problem:

$$
\begin{aligned}
\frac{d S}{d t}=\mu N(1-p)-(\mu+r(t)) S+\delta(N-S) & \\
& S(0)=S_{0} \in \mathbb{R}_{+} .
\end{aligned}
$$

From $[5,14]$, the equation above admits a unique positive $L T$-periodic solution given by

$$
\begin{aligned}
\widehat{S}(t) & =e^{-\int_{0}^{t}(\mu+r(s)+\delta) d s}(\widehat{S}(0) \\
& \left.+N(\mu(1-p)+\delta) \int_{0}^{t} e^{\int_{0}^{s}(\mu+r(\xi)+\delta) d \xi} d s\right),
\end{aligned}
$$

where

$$
\widehat{S}(0)=\frac{N(\mu(1-p)+\delta) \int_{0}^{L T} e^{\int_{0}^{s}(\mu+r(\xi)+\delta) d \xi} d s}{e^{\int_{0}^{L T}(\mu+r(s)+\delta) d s}-1} .
$$

Therefore, $(\widehat{S}(t), 0,0)$ is a disease-free periodic solution of (7); moreover, from [5] we have that $\widehat{S}(t) \leq N$; therefore, $(\widehat{S}(t), 0,0)$ lives in $X$.

Using the notation of [9], we sort the compartments so that the first two compartments correspond to infected individuals. Let $x=(E, I, S)$ and define

(i) $\mathscr{F}_{i}$ : the rate of new infection in compartment $i$,

(ii) $\mathscr{V}_{i}^{+}$: the rate of individuals into compartment $i$ by other means,

(iii) $\mathscr{V}_{i}^{-}$: the rate of individuals transfer out of compartment $i$.

System can be written as

$$
x^{\prime}(t)=\left(\begin{array}{c}
\beta(t) S f(I)-(\mu+\sigma) E \\
\sigma E-(\mu+\gamma) I \\
\mu N(1-p)-\beta(t) S f(I)-(\mu+r(t)) S+\delta(N-S-E-I)
\end{array}\right)=\mathscr{F}-\mathscr{V},
$$

where $\mathscr{V}=\mathscr{V}^{-}-\mathscr{V}^{+}$,

$$
\mathscr{F}=\left(\begin{array}{c}
\beta(t) S f(I) \\
0 \\
0
\end{array}\right)
$$$$
\mathscr{V}^{-}=\left(\begin{array}{c}
(\mu+\sigma) E \\
(\mu+\gamma) I \\
\beta(t) S f(I)+\delta(S+E+I)+(\mu+r(t)) S
\end{array}\right)
$$

Linearizing system (12) around the disease-free solution, we obtain the matrix of partial derivatives $J(0,0, \widehat{S})=$ $D \mathscr{F}(0,0, \widehat{S})-D \mathscr{V}(0,0, \widehat{S})$, where 


$$
\begin{aligned}
& D \mathscr{F}(0,0, \widehat{S})=\left(\begin{array}{ccc}
0 & \beta(t) \widehat{S} f^{\prime}(0) & 0 \\
0 & 0 & 0 \\
0 & 0 & 0
\end{array}\right) \\
& D \mathscr{V}(0,0, \widehat{S}) \\
& =\left(\begin{array}{ccc}
\mu+\sigma & 0 & 0 \\
-\sigma & \mu+\gamma & 0 \\
\delta & \beta(t) \widehat{S} f^{\prime}(0)+\delta & \delta+\mu+r(t)
\end{array}\right) .
\end{aligned}
$$

Using Lemma 1 of [9], we part $D \mathscr{F}$ and $D \mathscr{V}$ and set

$$
\begin{aligned}
F(t) & =\left(\begin{array}{cc}
0 & \beta(t) \widehat{S} f^{\prime}(0) \\
0 & 0
\end{array}\right), \\
V(t) & =\left(\begin{array}{cc}
\mu+\sigma & 0 \\
-\sigma & \mu+\gamma
\end{array}\right) .
\end{aligned}
$$

For a compartmental epidemiological model based on an autonomous system, the basic reproduction number is determined by the spectral radius of the next-generation matrix $F V^{-1}$ (which is independent of time) [9]. The definition of basic reproduction number for nonautonomous systems has been studied for multiple authors; see, for example, [12, 13]. Particularly, Wang and Zhao in [13] extended the work of [9] to include epidemiological models in periodic environments. They introduced the next infection operator $\mathscr{L}: C_{L T} \rightarrow C_{L T}$ given by

$$
\begin{array}{r}
(\mathscr{L} \phi)(t)=\int_{0}^{\infty} Y(t, t-a) F(t-a) \phi(t-a) d a, \\
\forall t \in \mathbb{R}, \phi \in C_{L T},
\end{array}
$$

where $C_{L T}$ is the ordered Banach space of all $L T$ periodic functions from $\mathbb{R}$ to $\mathbb{R}^{2}$, which is equipped with the maximum norm. $\phi(s) \in C_{L T}$ is the initial distribution of infectious individuals in this periodic environment, and $Y(t, s), t \geq s$ is the evolution operator of the linear periodic system:

$$
\frac{d y}{d t}=-V(t) y
$$

meaning that, for each $s \in \mathbb{R}$, the $2 \times 2$ matrix $Y$ satisfies

$$
\frac{d Y(t, s)}{d t}=-V(t) Y(t, s), \quad \forall t \geq s, Y(s, s)=I_{2 \times 2} .
$$

$\mathscr{L} \phi$ is the distribution of accumulative new infections at time $t$ produced by all those infected individuals $\phi(s)$ introduced before $t$, with kernel $K(t, a)=Y(t, t-a) F(t-a)$. The coefficient $K_{i, j}(t, a)$ in row $i$ and column $j$ represents the expected number of individuals in compartment $I_{i}$ that one individual in compartment $I_{j}$ generates at the beginning of an epidemic per unit time at time $t$ if it has been in compartment $I_{j}$ for $a$ units of time, with $I_{1}=E, I_{2}=I$ [16].

Let $r_{0}>0, r_{0}$ is an eigenvalue of $\mathscr{L}$ if there is a nonnegative eigenfunction $v(t) \in C_{L T}$ such that

$$
\mathscr{L} v=r_{0} v
$$

Therefore, the basic reproduction number is defined as

$$
\mathscr{R}_{0}:=\rho(\mathscr{L}),
$$

the spectral radius of $\mathscr{L}$. The basic reproduction number can be evaluated by several numerical methods and approximations [15-17]; in Section 4 we discuss this topic.

\section{The Threshold Dynamics of $R_{0}$}

\subsection{Disease Extinction}

Theorem 2. Let $\mathscr{R}_{0}$ be defined as (20); then the disease-free periodic solution $(\widehat{S}(t), 0,0)$ is asymptotically stable if $\mathscr{R}_{0}<1$ and unstable if $\mathscr{R}_{0}>1$.

Proof. We use Theorem 2.2 of [13] and check conditions (A1)-(A7). Conditions (A1)-(A5) are clearly satisfied from the definitions of $\mathscr{F}$ and $\mathscr{V}$ given in Section 2 . We prove only conditions (A6) and (A7). Define

$$
M(t):=-(\mu+r(t)+\delta)
$$

and let $\Phi_{M}(t)$ be the monodromy matrix of system

$$
\frac{d z}{d t}=M(t) z
$$

(A6) $\rho\left(\Phi_{M}(L T)\right)<1$. Let $\Psi_{M}$ be a fundamental matrix for system $d z / d t=M(t) z$, with $M$ defined as before and $L T$ periodic; the monodromy matrix $\Phi_{M}(L T)$ is given by $\Phi_{M}(L T)=\Psi_{M}^{-1}(0) \Psi_{M}(L T)$. The general solution of (22) is

$$
z(t)=K \exp \left(-\int_{0}^{t}(\mu+r(s)+\delta) d s\right),
$$

so $\Psi_{M}=\exp \left(-\int_{0}^{t}(\mu+r(s)+\delta) d s\right)$ and $\Psi_{M}^{-1}=$ $\exp \left(\int_{0}^{t}(\mu+r(s)+\delta) d s\right)$. Note that $\Psi_{M}^{-1}(0)=1$, so $\Phi_{M}(L T)=\Psi_{M}(L T)$ and

$$
\Phi_{M}(L T)=\exp \left(-\int_{0}^{L T}(\mu+r(s)+\delta) d s\right) .
$$

Due to the fact that $\Phi_{M}(L T)$ is a constant, its eigenvalue is itself and $\rho\left(\Phi_{M}(L T)\right)<1$ for $\mu, \delta, r(s)>0$.

(A7) $\rho\left(\Phi_{-V}(L T)\right)<1$. Solving the system $d z / d t=-V(t) z$, we arrive at the general solution

$$
z(t)=c_{1}\left(\begin{array}{c}
\frac{\gamma-\sigma}{\sigma} \\
1
\end{array}\right) e^{-(\mu+\sigma) t}+c_{2}\left(\begin{array}{l}
0 \\
1
\end{array}\right) e^{-(\mu+\gamma) t}
$$

so

$$
\Psi_{-V}(t)=\left(\begin{array}{cc}
\frac{\gamma-\sigma}{\sigma} e^{-(\mu+\sigma) t} & 0 \\
e^{-(\mu+\sigma) t} & e^{-(\mu+\gamma) t}
\end{array}\right) .
$$


Computing $\Phi_{-V}(L T)=\Psi_{-V}^{-1}(0) \Psi_{-V}(L T)$, we have

$$
\Phi_{-V}(L T)=\left(\begin{array}{cc}
e^{-(\mu+\sigma) L T} & 0 \\
0 & e^{-(\mu+\gamma) L T}
\end{array}\right) .
$$

Clearly, $\rho\left(\Phi_{-V}(L T)\right)=\max \left\{e^{-(\mu+\sigma) L T}, e^{-(\mu+\gamma) L T}\right\}<1$ for $\mu, \gamma, \sigma>0$.

Note 1. Due to the fact that $\Psi_{A}$ is a fundamental solution of a periodic system, we can always choose it such that $\Psi(0)=I$, so the monodromy matrix satisfies $\Phi_{A}(L T)=\Psi_{A}(L T)$. This property is used in further analysis.

In order to prove the global stability of the disease-free periodic solution, we enunciate some useful definitions and some lemmas.

Let $A(t)$ be continuous, cooperative, irreducible, and $\omega$ periodic $k \times k$ matrix function, and $\Psi_{A}(t)$ the fundamental matrix of system $x^{\prime}(t)=A(t) x(t)$. Denote by $\rho\left(\Psi_{A}(\omega)\right)$ the spectral radius of $\Psi_{A}(\omega)$.

Lemma 3. Let $p=(1 / \omega) \ln \rho\left(\Psi_{A}(\omega)\right)$. Then there exists a positive, $\omega$-periodic function $v(t)$ such that $e^{p t} v(t)$ is a solution of $x^{\prime}(t)=A(t) x(t)$ (see proof in Lemma 2.1 of [18]).

Lemma 4. Function $f(I)$ of model (1) satisfies $f(I) \leq f^{\prime}(0) I$, $\forall I \geq 0$.

Proof. Using assumptions on function $f$, we have

$$
\frac{d}{d I}\left(\frac{f(I)}{I}\right)=\frac{I f^{\prime}(I)-f(I)}{I^{2}} \leq 0,
$$

so function $f(I) / I$ decreases $\forall I>0$ and then $f(I) / I \leq$ $\lim _{I \rightarrow 0^{+}}(f(I) / I)=f^{\prime}(0)$.

Lemma 5. Let $(S(t), E(t), I(t))$ be a solution of system (7) with initial conditions $\left(S_{0}, E_{0}, I_{0}\right) \geq 0$, and $(\widehat{S}(t), 0,0)$ the diseasefree periodic solution of (7); then

$$
\limsup _{t \rightarrow \infty}(S(t)-\widehat{S}(t)) \leq 0
$$

Proof. Proof is similar to Lemma 4.1 of [14]. $S(t)$ satisfies the first equation of system (7); then

$$
\begin{aligned}
\frac{d S}{d t}= & \mu N(1-p)-\beta(t) S f(I)-(\mu+r(t)) S \\
& +\delta(N-S-E-I) \\
\leq & N(\mu(1-p)+\delta)-(\mu+r(t)+\delta) S .
\end{aligned}
$$

Let $X(t)=S(t)-\widehat{S}(t)$; then

$$
\begin{aligned}
\frac{d X}{d t} & =(\mu+r(t)+\delta)(\widehat{S}-S)-\beta(t) S f(I)-\delta(E+I) \\
& \leq-(\mu+r(t)+\delta) X
\end{aligned}
$$

Using Gronwall's inequality $X(t) \leq X(0) e^{-\int_{0}^{t}(\mu+r(s)+\delta) d s}$,

$$
\begin{aligned}
S(t)-\widehat{S}(t) & \leq(S(0)-\widehat{S}(0)) e^{-\int_{0}^{t}(\mu+r(s)+\delta) d s} \\
& =(S(0)-\widehat{S}(0)) e^{-(\mu+\delta) t} e^{-\int_{0}^{t} r(s) d s} .
\end{aligned}
$$

Taking limits in both sides, we obtain that $\lim _{\sup } \operatorname{su\infty }_{t \rightarrow \infty} S(t)-$ $\widehat{S}(t) \leq 0$.

Now, we are able to enunciate our theorem for global stability of disease-free periodic solution.

Theorem 6. The disease-free periodic solution $(\widehat{S}(t), 0,0)$ of system (7) is globally asymptotically stable if $\mathscr{R}_{0}<1$.

Proof. From Theorem 2 we have that $(\widehat{S}(t), 0,0)$ is unstable for $\mathscr{R}_{0}>1$ and asymptotically stable for $\mathscr{R}_{0}<1$, so it is sufficient to prove that any solution $(S(t), E(t), I(t))$ with nonnegative initial conditions $\left(S_{0}, E_{0}, I_{0}\right)$ approaches $(\widehat{S}, 0,0)$ as $t$ tends to infinity.

Let $\epsilon>0$; from Lemma 5 we have

$$
\begin{aligned}
\limsup _{t \rightarrow \infty}(S(t)-\widehat{S}(t)) & =\lim _{t \rightarrow \infty} \sup _{\tau \geq t}(S(\tau)-\widehat{S}(\tau))=L \\
& \leq 0,
\end{aligned}
$$

so there exists a $N>0$ such that for all $t_{1}>N$

$$
-\epsilon<\sup _{t \geq t_{1}}(S(t)-\widehat{S}(t))-L<\epsilon,
$$

which implies that $\sup _{t \geq t_{1}}(S(t)-\widehat{S}(t))<\epsilon+L \leq \epsilon$. Then, from the definition of supremum, we have that for all $t>t_{1}$

$$
S(t)-\widehat{S}(t) \leq \sup _{t \geq t_{1}}(S(t)-\widehat{S}(t))<\epsilon .
$$

Then, we have proved that for all $\epsilon>0$ we can find a $t_{1}>0$ such that $S(t)<\epsilon+\widehat{S}(t)$ for all $t>t_{1}$.

Now, using Lemma 4 , for $\epsilon>0$ we can find a $t_{1}>0$ such that for $t>t_{1}$

$$
\begin{aligned}
\frac{d E}{d t} & =\beta(t) S f(I)-(\mu+\sigma) E \\
& \leq \beta(t) S(t) f^{\prime}(0) I-(\mu+\sigma) E(t) \\
& <\beta(t) f^{\prime}(0)(\widehat{S}(t)+\epsilon) I(t)-(\mu+\sigma) E(t) .
\end{aligned}
$$

We consider the following perturbed subsystem:

$$
\begin{aligned}
& \frac{d \bar{E}}{d t}=\beta(t) f^{\prime}(0)(\widehat{S}+\epsilon) \bar{I}-(\mu+\sigma) \bar{E} \\
& \frac{d \bar{I}}{d t}=\sigma \bar{E}-(\mu+\gamma) \bar{I}
\end{aligned}
$$

which can be rewritten as

$$
\begin{aligned}
\left(\frac{d \bar{E}}{d t}, \frac{d \bar{I}}{d t}\right)^{T}= & (F(t)-V(t))(\bar{E}, \bar{I})^{T} \\
& +\epsilon H(t)(\bar{E}, \bar{I})^{T},
\end{aligned}
$$


with $F(t), V(t)$ defined in (15) and

$$
H(t)=\left(\begin{array}{cc}
0 & \beta(t) f^{\prime}(0) \\
0 & 0
\end{array}\right) .
$$

Matrix $(F-V+\epsilon H)(t)$ is $L T$-periodic, cooperative, irreducible, and continuous. Using Lemma 3 , if $q=$ $(1 / L T) \ln \rho\left(\Psi_{F-V+\epsilon H}(L T)\right)$, then there exists a positive and $L T$-periodic function $v(t)=\left(v_{1}(t), v_{2}(t)\right)^{T}$ such that $e^{q t} v(t)$ is solution of system (38). Note that for all $k>0$, function $k e^{q\left(t-t_{i}\right)} v\left(t-t_{i}\right)$ is also a solution of system (38) with initial condition $k v(0)$ at $t=t_{i}$.

Choose a $\bar{t}>t_{1}$ and $\alpha_{1}>0$ such that $(E(\bar{t}), I(\bar{t}))^{T} \leq$ $\alpha_{1} v(0)$; then from (37) we have that

$$
\left(\frac{d E}{d t}, \frac{d I}{d t}\right)^{T} \leq(F-V)(E, I)^{T}+\epsilon H(E, I)^{T},
$$

and using a comparison principle (see, e.g., [19] Theorem B.1), we have $(E(t), I(t))^{T} \leq \alpha_{1} e^{q(t-\bar{t})} v(t-\bar{t})$ for all $t>\bar{t}$.

From Theorem 2.2 of [13], $\mathscr{R}_{0}<1$ iff $\rho\left(\Phi_{F-V}(L T)\right)<$ 1. By the continuity of the spectrum for matrices (see [20], Section II.5.8), we can choose $\epsilon>0$ small enough so that $\rho\left(\Phi_{F-V+\epsilon H}(L T)\right)<1$ and then $q<0$ (see Note 1 ). Thus, using positivity of solutions and comparison,

$$
0 \leq \lim _{t \rightarrow \infty} E(t) \leq \lim _{t \rightarrow \infty} \alpha_{1} e^{q(t-\bar{t})} v_{1}(t-\bar{t})=0 .
$$

And similarly for $I$, we obtain that

$$
\begin{aligned}
& \lim _{t \rightarrow \infty} E(t)=0 \\
& \lim _{t \rightarrow \infty} I(t)=0 .
\end{aligned}
$$

We need only to prove that $S(t)$ approaches $\widehat{S}$. At diseasefree periodic solution $\widehat{R}(t)=N-\widehat{S}(t)$, where $\widehat{R}$ satisfies

$$
\frac{d \widehat{R}}{d t}=\mu N p+r(t) \widehat{S}-(\mu+\delta) \widehat{R}
$$

Thus, $R(t)=N-S(t)-E(t)-I(t)$ satisfies

$$
\frac{d(R-\widehat{R})}{d t}=r(t)(S-\widehat{S})+\gamma I-(\mu+\delta)(R-\widehat{R}) .
$$

Let $\epsilon_{1}>0$ be arbitrary and $r_{\max }=\max _{u \in[0, L T]} r(u)$. Due to (43) we can find a $t_{2}>0$ such that $I(t)<\epsilon_{1}$ for $t>t_{2}$; moreover, we can find a $t_{3}>0$ such that $S(t) \leq \widehat{S}(t)+\epsilon_{1}$ for $t>t_{3}$. Then, let $t_{4}=\max \left\{t_{2}, t_{3}\right\}$; we have for $t>t_{4}$

$$
\frac{d(R-\widehat{R})}{d t} \leq\left(r_{\max }+\gamma\right) \epsilon_{1}-(\mu+\delta)(R-\widehat{R}) .
$$

Multiplying in both sides by $e^{(\mu+\delta) t}$ and integrating from $t_{4}$ to $t$, we obtain

$$
\begin{aligned}
(R-\widehat{R}) \leq & (R-\widehat{R})\left(t_{4}\right) e^{-(\mu+\delta)\left(t-t_{4}\right)} \\
& +\frac{\epsilon_{1}\left(r_{\max }+\gamma\right)}{\mu+\delta}\left(1-e^{-(\mu+\delta)\left(t-t_{4}\right)}\right) .
\end{aligned}
$$

So, $\lim _{\sup _{t \rightarrow \infty}}(R-\widehat{R})(t) \leq \epsilon_{1}\left(r_{\max }+\gamma\right) /(\mu+\delta)$, where $\epsilon_{1}\left(r_{\max }+\gamma\right) /(\mu+\delta)$ is arbitrarily small. Then lim $\sup _{t \rightarrow \infty}(R-$ $\widehat{R})(t) \leq 0$, and using similar arguments for $S$ and $\epsilon_{2}>0$, we can find a $t_{5}>0$ with $R(t) \leq \widehat{R}(t)+\epsilon_{2} / 2$ for $t>t_{5}$. Also, from (43), we can find $t_{6}>0$ with $E(t)+I(t)<\epsilon_{2} / 2$ for $t>t_{6}$, so, for $t>\max \left\{t_{5}, t_{6}\right\}$, we have

$$
\begin{aligned}
S(t) & =N-E(t)-I(t)-R(t) \geq N-\widehat{R}(t)-\epsilon_{2} \\
& =\widehat{S}(t)-\epsilon_{2} .
\end{aligned}
$$

Or, equivalently, $S(t)-\widehat{S}(t) \geq-\epsilon_{2}$, with $\epsilon_{2}$ being arbitrarily small, and this implies that $\lim _{i n f}(S-\widehat{S})(t) \geq$ 0 . We conclude by comparison and using Lemma 5 that $\lim _{t \rightarrow \infty} S(t)=\widehat{S}(t)$, completing the proof.

Theorem 6 shows that disease will completely disappear as long as $\mathscr{R}_{0}<1$. Thus, reducing and keeping $\mathscr{R}_{0}$ below the unity would be sufficient to eradicate infection, even in a periodic environment and a general incidence rate.

3.2. Disease Persistence. Uniform persistence is an important concept in population dynamics, since it characterizes the long-term survival of some or all interacting species in an ecosystem [21].

In this section we consider the dynamics of the periodic model when $\mathscr{R}_{0}>1$. We will show that actually $\mathscr{R}_{0}$ is a threshold parameter for the extinction and the uniform persistence of the disease. Our results are inspired by $[5,15$, $18,22]$.

Let $P: X \rightarrow X$ be the Poincaré map associated with system (7); that is,

$$
P\left(x_{0}\right)=\phi\left(L T, x_{0}\right), \quad \forall x_{0} \in X,
$$

where $X$ is defined in (8) and $\phi\left(t, x_{0}\right)$ is the unique solution of system (7) with $\phi\left(0, x_{0}\right)=x_{0}$. We define the following sets:

$$
X_{0}:=\{(S, E, I) \in X: E>0, I>0\},
$$

$$
\partial X_{0}:=X \backslash X_{0}
$$

Note that $\partial X_{0}$ is not the boundary of $X_{0}$, but it is a standard notation of persistence theory.

Lemma 7. Set $X_{0}$ is positively invariant under system (7).

Proof. Let $x_{0}=\left(S_{0}, E_{0}, I_{0}\right) \in X_{0}$, that is, $E_{0}>0, I_{0}>0$, and let

$$
\phi\left(t, x_{0}\right)=(S(t), E(t), I(t))
$$

be the solution of $(7)$ with $\phi\left(0, x_{0}\right)=x_{0}$. Due to nonnegativity of solutions and assumptions on function $\beta(t)$ and $f(I)$, we have

$$
\frac{d E}{d t}=\beta(t) S f(I)-(\mu+\sigma) E \geq-(\mu+\sigma) E,
$$


Using a comparison theorem (see, e.g., [19] Appendix B.1), we have for all $t>0$

$$
E(t) \geq K e^{-(\mu+\sigma) t}>0, \quad \text { with } K=E(0)>0 .
$$

Similarly,

$$
\frac{d I}{d t}=\sigma E-(\mu+\gamma) I \geq-(\mu+\gamma) I
$$

so,

$$
I(t) \geq I(0) e^{-(\mu+\gamma)} t>0, \quad \forall t>0 .
$$

Therefore, $\phi\left(t, x_{0}\right)$ remains on $X_{0}$ for all $t>0$.

To use persistence theory developed in [21], we show that

$$
M_{\partial}=\{(S, 0,0): S \geq 0\} \text {, }
$$

where

$$
\begin{aligned}
M_{\partial} & :=\left\{\left(S_{0}, E_{0}, I_{0}\right) \in \partial X_{0}: P^{m}\left(S_{0}, E_{0}, I_{0}\right) \in \partial X_{0}, \forall m\right. \\
& \geq 0\} .
\end{aligned}
$$

Let $x_{0}=\left(S_{0}, 0,0\right) \in X$ and $(S(t), E(t), I(t))$ be the solution that passes through that initial condition. We have that $\phi\left(t, x_{0}\right)=\left(S_{1}(t), 0,0\right)$, with $S_{1}(t)$ being a solution of (9) and $S_{1}(0)=S_{0}$ being a solution that satisfies the initial condition. By uniqueness of solutions we have $E(t)=0=$ $I(t) \forall t \geq 0$, so $x_{0}$ lives on $M_{\partial}$.

Now, if $x_{0} \in M_{\partial}$, we want $x_{0}=\left(S_{0}, 0,0\right)$. We prove an equivalent sentence: if $x_{0} \in \partial X_{0} \backslash\{(S, 0,0): S \geq 0\}$, then it does not belong to $M_{\partial}$. Consider an initial point $x_{0}=$ $\left(S_{0}, E_{0}, I_{0}\right) \in \partial X_{0} \backslash\{(S, 0,0): S \geq 0\}$; then $E_{0}>0, I_{0}=0$, or $E_{0}=0, I_{0}>0$. Suppose that $E>0$ and $I_{0}=0$; then $\phi\left(t, x_{0}\right)$ holds

$$
\frac{d I}{d t}(0)=\sigma E(0)>0
$$

By continuity of $E(t)$ and sign of derivative of $I$, we have that, for small $0<t \ll 1, E(t)>0, I(t)>0$, so, for $0<t \ll 1, \phi\left(t, x_{0}\right) \in X_{0}$. Using invariance of $X_{0}$ (Lemma 7) we have $\phi\left(t, x_{0}\right) \in X_{0}$ for all $t>1$. Finally, for a $m>0$ such that $m L T>1$, we have $P^{m}\left(x_{0}\right)=\phi\left(m L T, x_{0}\right) \in X_{0}$ and this implies (56). By the existence of a disease-free periodic solution (proved in Section 2), it is clear that there is one fixed point of $P$ in $M_{\partial}$ given by $M_{0}=(\widehat{S}(0), 0,0)([23])$.

Now, we are in a position to introduce the following result of uniform persistence of the disease.

Theorem 8. Let $\mathscr{R}_{0}>1$; then there exists an $\epsilon>0$ such that any solution $(S(t), E(t) I(t))$ of (7) with initial values $(S(0), E(0), I(0)) \in X_{0}$ satisfies

$$
\begin{aligned}
& \liminf _{t \rightarrow \infty} E(t) \geq \epsilon, \\
& \liminf _{t \rightarrow \infty} I(t) \geq \epsilon .
\end{aligned}
$$

Proof. We first prove that $P$ is uniformly persistent (see Definition 1.3.2 from [21]) with respect to $\left(X_{0}, \partial X_{0}\right)$, because this implies that the solution of (7) is uniformly persistent with respect to $\left(X_{0}, \partial X_{0}\right)$ (see [21], Theorem 3.1.1). Clearly, $X_{0}$ is relatively open in $X$, so $\partial X_{0}$ is relatively closed.

Define

$$
W^{s}:=\left\{x_{0} \in X_{0}: \lim _{m \rightarrow \infty}\left\|P^{m}\left(x_{0}\right)-M_{0}\right\|=0\right\} ;
$$

we show that $W^{s}\left(M_{0}\right) \cap X_{0}=\emptyset$.

By Theorem 2.2 of [13], $\mathscr{R}_{0}>1$ if and only if $r\left(\Psi_{F-V}(L T)\right)>1$. Choose an $\eta>0$ small enough with the property $\widehat{S}(t)-\eta>0, \forall t>0$ (see Appendix A). For $\alpha>0$, let us consider the following perturbed equation:

$$
\begin{aligned}
\frac{d \bar{S}}{d t}= & N(\mu(1-p)+\delta)-2 \delta \alpha \\
& -\left(\beta(t) f^{\prime}(0) \alpha+\mu+r(t)+\delta\right) \bar{S}
\end{aligned}
$$

System above admits a unique positive $L T$-periodic solution of the form

$$
\begin{gathered}
\widehat{S}(t, \alpha)=e^{-\int_{0}^{t}\left(\beta(s) f^{\prime}(0) \alpha+\mu+r(s)+\delta\right) d s}(\widehat{S}(0, \alpha) \\
+(N \mu(1-p)+N \delta-2 \delta \alpha) \\
\left.\cdot \int_{0}^{t} e^{\int_{0}^{s}\left(\beta(\xi) f^{\prime}(0) \alpha+\mu+r(\xi)+\delta\right) d \xi} d s\right)
\end{gathered}
$$

whit $\widehat{S}(t, 0)=\widehat{S}(t)$, which is globally attractive for all solutions of (61) (see Appendix B for proof), and with

$$
\begin{aligned}
& \widehat{S}(0, \alpha) \\
& =\frac{(N \mu(1-p)+N \delta-2 \delta \alpha) \int_{0}^{L T} e^{\int_{0}^{s}\left(\beta(\xi) f^{\prime}(0) \alpha+\mu+r(\xi)+\delta\right) d \xi} d s}{e^{\int_{0}^{L T}\left(\beta(s) f^{\prime}(0) \alpha+\mu+r(s)+\delta\right) d s}-1} .
\end{aligned}
$$

Since $\widehat{S}(0, \alpha)$ is continuous in $\alpha$, for all $\epsilon>0$ there is a $\delta>$ 0 such that for $|\alpha|<\delta$ we have $|\widehat{S}(0, \alpha)-\widehat{S}(0,0)|<\epsilon$. Moreover, by continuity of solutions with respect to initial values we can find for all $\bar{\eta}>0$ an $\bar{\epsilon}>0$ such that if $|\widehat{S}(0, \alpha)-\widehat{S}(0,0)|<\bar{\epsilon}$, then

$$
|\widehat{S}(t, \alpha)-\widehat{S}(t, 0)|<\bar{\eta}
$$

Therefore, for $\eta$ established before, we can find $\alpha$ small enough such that $\widehat{S}(t, \alpha)>\widehat{S}(t)-\eta, \forall t>0$.

Again, by continuity of solutions with respect to initial values, for this small $\alpha>0$, there exists a $\delta>0$ such that for all $\left(S_{0}, E_{0}, I_{0}\right) \in X_{0}$ with $\left\|\left(S_{0}, E_{0}, I_{0}\right)-M_{0}\right\| \leq \delta$ we have $\left\|\phi\left(t,\left(S_{0}, E_{0}, I_{0}\right)\right)-\phi\left(t, M_{0}\right)\right\|<\alpha, \forall t \in[0, L T]$.

We now claim that

$$
\begin{aligned}
\limsup _{m \rightarrow \infty}\left\|P^{m}\left(S_{0}, E_{0}, I_{0}\right)-M_{0}\right\| \geq \delta, & \\
& \forall\left(S_{0}, E_{0}, I_{0}\right) \in X_{0} .
\end{aligned}
$$


By contradiction, suppose that

$$
\begin{aligned}
\limsup _{m \rightarrow \infty} \| P^{m}\left(S_{0}, E_{0}, I_{0}\right)- & M_{0} \|<\delta, \\
& \text { for some }\left(S_{0}, E_{0}, I_{0}\right) \in X_{0} .
\end{aligned}
$$

Without loss of generality, we can assume that $\left\|P^{m}\left(\mathrm{~S}_{0}, E_{0}, I_{0}\right)-M_{0}\right\|<\delta$ for all $m \geq 0$ (see Appendix $\mathrm{C}$ ). From the discussion above, $\left\|\phi\left(t, P^{m}\left(S_{0}, E_{0}, I_{0}\right)\right)-\phi\left(t, M_{0}\right)\right\|<$ $\alpha, \forall m \geq 0$ and $t \in[0, L T]$.

For any $t \geq 0$, let $t=m L T+t_{1}$, where $t_{1} \in[0, L T)$ and $m=[t / L T]$ is the greatest integer less than or equal to $t / L T$. Then, we get

$$
\begin{aligned}
& \phi\left(t,\left(S_{0}, E_{0}, I_{0}\right)\right)-\phi\left(t, M_{0}\right) \\
& \quad=\phi\left(t_{1}, P^{m}\left(S_{0}, E_{0}, I_{0}\right)\right)-\phi\left(t, M_{0}\right)<\alpha .
\end{aligned}
$$

If we set $\phi\left(t,\left(S_{0}, E_{0}, I_{0}\right)\right)=(S(t), E(t), I(t))$, then we have $E(t) \leq \alpha, I(t) \leq \alpha, \forall t \geq 0$, and from the first equation of (7) and Lemma 4 we arrive at

$$
\begin{aligned}
\frac{d S}{d t} \geq & N(\mu(1-p)+\delta)-2 \delta \alpha \\
& -\left(\beta(t) f^{\prime}(0) \alpha+\mu+r(t)+\delta\right) \bar{S}
\end{aligned}
$$

which is exactly the equation in (61). Since the unique periodic solution of (61) is globally attractive, we have for $\bar{S}(t, \alpha)$ solution of $(61)$ that $\lim _{t \rightarrow \infty} \bar{S}(t, \alpha)=\widehat{S}(t, \alpha)$. So for $\eta$ given before, there exists $T>0$ such that for all $t \geq T$

$$
|\bar{S}(t, \alpha)-\widehat{S}(t, \alpha)|<\eta
$$

or equivalently $\bar{S}(t, \alpha)>\widehat{S}(t, \alpha)-\eta$. Moreover, from previous analysis, $\widehat{S}(t, \alpha)-\eta>\widehat{S}(t)-\eta$; therefore, using comparison principle on (68) we arrive at

$$
S(t) \geq \widehat{S}(t)-\eta
$$

for $t>T$.

We have $E(t), I(t) \leq \alpha$, and $\alpha$ is fixed small, so we can take $\alpha<\epsilon^{*}$ and use assumption (A5) in Introduction (see Appendix D) to obtain

$$
\left(\begin{array}{l}
\frac{d E}{d t} \\
\frac{d I}{d t}
\end{array}\right) \geq(F-V-\eta H-\alpha K)(E, I)^{T},
$$

where $F, V$ are defined in (15), $H$ is defined in (40), and

$$
K=\left(\begin{array}{cc}
0 & -\frac{1}{2} \beta(t) f^{\prime \prime}(0)[\widehat{S}-\eta] \\
0 & 0
\end{array}\right)
$$

By Theorem 2.2 of [13], we have $\mathscr{R}_{0}>1$ iff $\rho\left(\Phi_{F-V}(L T)\right)>$ 1. By continuity of spectrum (see [20] Section II), we can find $\alpha, \epsilon$ such that

$$
\rho\left(\Phi_{F-V-\eta H-\alpha K}\right)>1
$$

Consider the auxiliary system

$$
\left(\begin{array}{c}
\frac{d E_{2}}{d t} \\
\frac{d I_{2}}{d t}
\end{array}\right)=(F-V-\eta H-\alpha K)\left(E_{2}, I_{2}\right)^{T}
$$

then, using Lemma 3 there exists a solution of (71) with the form $e^{p_{2} t} v_{2}(t)$, with $p_{2}=(1 / L T) \ln \left(\rho\left(\Phi_{F-V-\eta H-\alpha K}(L T)\right)\right)>$ 0 . Choose a $t_{2}>T$ and a small number $\alpha_{2}>0$ such that $\left(E_{2}\left(t_{2}\right), I_{2}\left(t_{2}\right)\right)^{T} \geq \alpha_{2} v_{2}(0)$. Using comparison principle we get $(E(t), I(t)) \geq \alpha_{2} v_{2}\left(t-t_{2}\right) e^{p_{2}\left(t-t_{2}\right)}$, which implies $E(t) \rightarrow \infty$ and $I(t) \rightarrow \infty$. This leads to a contradiction.

The claim above shows that $P$ is weakly uniformly persistent with respect to $\left(X_{0}, \partial X_{0}\right)$. Note that $P$ has a global attractor $\widehat{S}(0)$ (see Lemma 5). It follows that $M_{0}$ is an isolated invariant set in $X, W^{s}\left(M_{0}\right) \cap X_{0}=\emptyset$. Every orbit in $M_{\partial}$ converges to $M_{0}$ and $M_{0}$ is acyclic. By the acyclicity theorem on uniform persistence for maps ([21] Theorem 1.3.1 and Remark 1.3.1), it follows that $P$ is uniformly persistent with respect to $\left(X_{0}, \partial X_{0}\right)$; that is, there exists $\epsilon>0$ such that any solution of (7) satisfies $\lim _{t \rightarrow \infty} E(t) \geq \epsilon, \lim _{t \rightarrow \infty} I(t) \geq \epsilon$.

\section{Numerical Simulations}

In this section we provide some numerical simulations to illustrate the results obtained in our theorems and compare them with previous results.

To improve previous models used in references, we use a particular function

$$
f(I)=\frac{I}{1+a I}, \quad a \geq 0,
$$

which includes the case $f(I)=I$ used in [5]. One can check that function (75) satisfies conditions (A1)-(A5). Using this function, system (7) is rewritten as

$$
\begin{aligned}
\frac{d S}{d t}= & \mu N(1-p)-\frac{\beta(t) S I}{1+a I}-(\mu+r(t)) S \\
& +\delta(N-S-E-I), \\
\frac{d E}{d t}= & \frac{\beta(t) S I}{1+a I}-(\mu+\sigma) E, \\
\frac{d I}{d t}= & \sigma E-(\mu+\gamma) I .
\end{aligned}
$$

Set an initial population $N=2,200,000$ and take time $t$ in years. Suppose $\mu=0.02$ per year, corresponding to an average human life time of 50 years. Following [5] take the parameters as follows: $\sigma=38.5$ per year, $\gamma=100$ per year, $p=0.85, \delta=$ 0 , and $a=1$. Choose the periodic transmission as $\beta(t)=\beta_{0}+$ $0.0002 \cos (2 \pi t)$, with $\beta_{0}$ being the transmission parameter, and the periodic vaccination rate $r(t)=0.1+0.004 \cos (2 \pi t)$. Both functions have period $L T=1$.

There exists multiple methods for computing the basic reproduction number, via numerical approximations, or finding a positive solution of the equation $\rho(W(L T, 0, \lambda))=1$ 


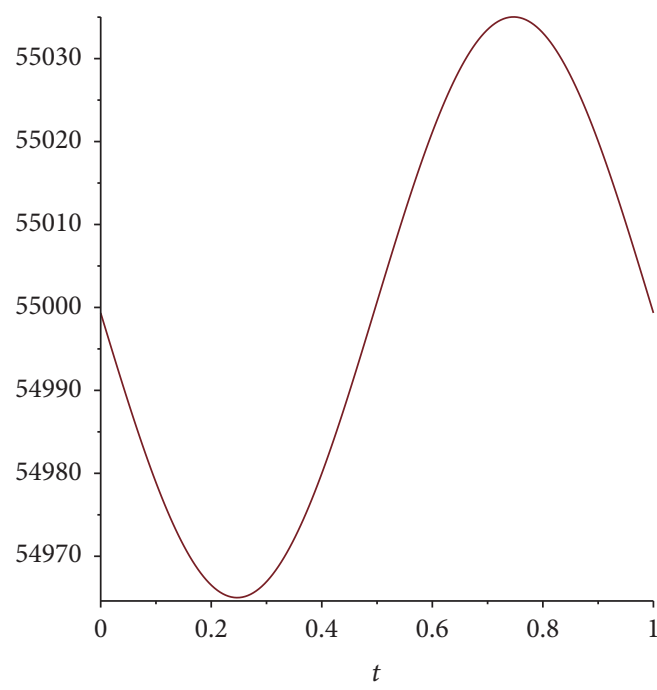

(a)

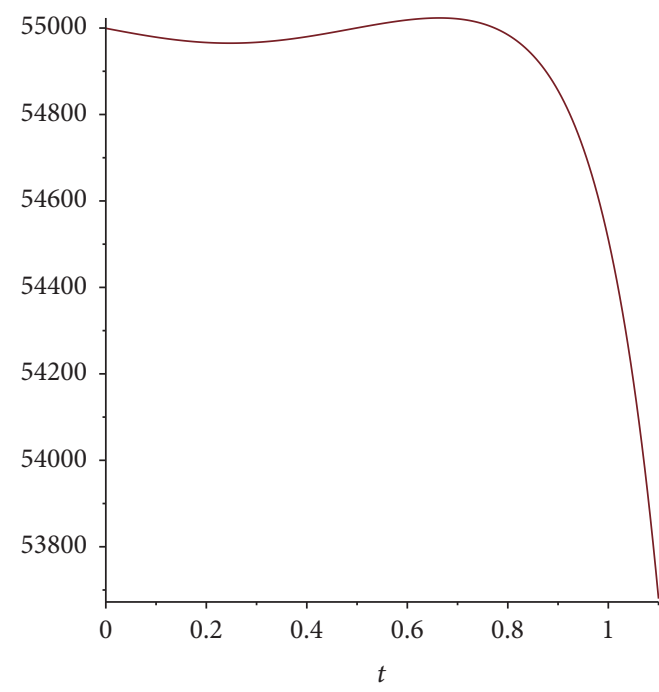

(b)

FIGURE 1: $S$ component of infection-free periodic solution. Time $t$ is given in years. (a) $\widehat{S}(t)$, because it is a periodic function of period 1 , is plotted only in $[0,1]$. (b) Taylor expansion of $\widehat{S}$ around $t=0$ of order $t^{10}$.

(see Theorem 2.1 of [13]). In order to compare our work with previous works, we approximate the basic reproduction number with its average value $\mathscr{R}_{0}^{T}$, used by several authors as the reproduction number (for example $[10,11]$ ), so define

$$
R_{0}^{T}=\rho\left([F] V^{-1}\right),
$$

where $V$ is given by (15) and

$$
[F]=\left(\begin{array}{cc}
0 & {[\beta][\hat{S}] f^{\prime}(0)} \\
0 & 0
\end{array}\right),
$$

with $[\beta],[\widehat{S}]$ being the average of functions $\beta, \widehat{S}$ defined as $[\beta]=(1 / L T) \int_{0}^{L T} \beta(t) d t, \widehat{S}=(1 / L T) \int_{0}^{L T} \widehat{S}(t) d t$. Computing each average, we obtain

$$
R_{0}^{T}=549.6702634 \beta_{0}
$$

so $R_{0}^{T}>1$ for $\beta_{0} \in(0.001819272510, \infty)$.

Following Theorem 2.1 of [13], to compute $\mathscr{R}_{0}$, let $W(t, s, \lambda), t \geq s$, be the evolution operator of the system

$$
\frac{d w}{d t}=\left(-V(t)+\frac{F(t)}{\lambda}\right) w
$$

that is, for each $\lambda \in(0, \infty), d W(t, s, \lambda) / d t=(-V(t)+$ $F(t) / \lambda) W(t, s, \lambda), \forall t \geq s$, and $W(s, s, \lambda)=I_{2 \times 2}$. With this operator, $\mathscr{R}_{0}>0$ is the unique solution of $\rho(W(L T, 0, \lambda))=1$.

Example 1. To illustrate our results, fix $\beta_{0}=0.0018$. Computing $R_{0}^{T}$, we have $R_{0}^{T}=0.9894064741$, which is a first approximation of $R_{0}$. To solve system (80) numerically, we substitute the terms of expression of $\widehat{S}(t)$ in (10):

$$
\begin{aligned}
\widehat{S}(t) & \\
& =e^{-0.1200000000 t-0.0006366197724 \sin (6.283185307 t)}(54999.33689 \\
& \left.+6600.0 \int_{0.0}^{t} e^{0.1200000000 s+0.0006366197724 \sin (6.283185307 s)} d s\right)
\end{aligned}
$$

The previous integral cannot be computed analytically, so we approach $\widehat{S}(t)$ using Taylor expansion around 0 (remember that we want so solve $\rho(W(L T, 0, \lambda))=1$, where $L T=1)$, so even when we cannot find an explicit expression for $\widehat{S}(t)$, the Taylor expansion is a good way to estimate it in $(0,1)$. It could be of interest to also use an approach of $\widehat{S}(t)$ around $t=1$ and compare the results with those obtained in the present work (see Section 5 for a discussion about this topic).

Setting an initial value $\lambda_{0}=0.98$ and letting $\lambda_{i}=\lambda_{0}+$ $i(0.0001)$, we solve system (80) numerically for each $\lambda_{i}$ (using initial conditions $w(0)=(1,0)$ and $w(0)=(0,1)$, to satisfy $\left.W(0,0)=I_{2 \times 2}\right)$, and compute $\rho_{1}=\rho\left(W\left(L T, 0, \lambda_{i}\right)\right)$ until $\rho_{1} \sim$ 1 . With previous process we arrive at $\rho_{1}=1.00120166209265$ for $\lambda=0.9872$ and $\rho_{1}=0.997826338969630$ for $\lambda=$ 0.9873; therefore $\mathscr{R}_{0} \in(0.9872,0.9873)$. Using a finer step size 0.0000001 to have more accuracy, we arrive at $\mathscr{R}_{0} \sim$ $0.9872355<1$.

Set initial values as $S(0)=1,500,000, E(0)=400,000$, $I(0)=40,000$, and $R(0)=N-(S(0)+E(0)+I(0))$.

There exist multiple numerical methods to compute and plot the solutions of nonautonomous differential equations; see, for example, the Adomian method, the homotopy analysis method, or the modified homotopy methods (see, e.g., $[24,25])$. For this work we use Matlab algorithms (ODE 45) to graph the solution of system (76) with these initial conditions. Figures 2 and 3 shows the results. We can see that $I(t), E(t)$ goes to zero, while $S(t), R(t)$ tend to stabilize; also $S(t)$ is tending to $\widehat{S}(t)$ with values between 54,000 and 56,000 (see Figure 1); this shows the results obtained in Theorem 6. 

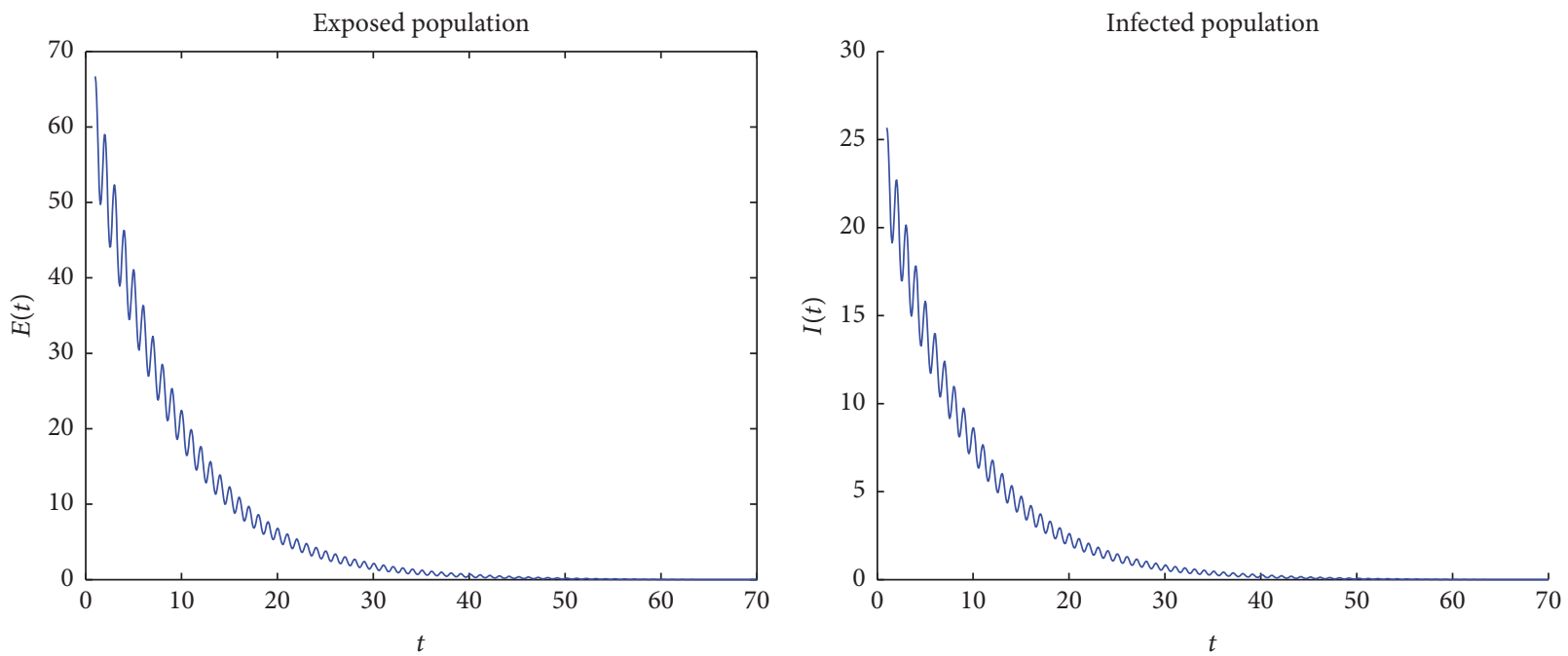

FIGURE 2: Solution of exposed and infected populations of SEIRS system when $\mathscr{R}_{0}<1$. We can see that both approach zero when time goes to infinity. Time $t$ is given in years.
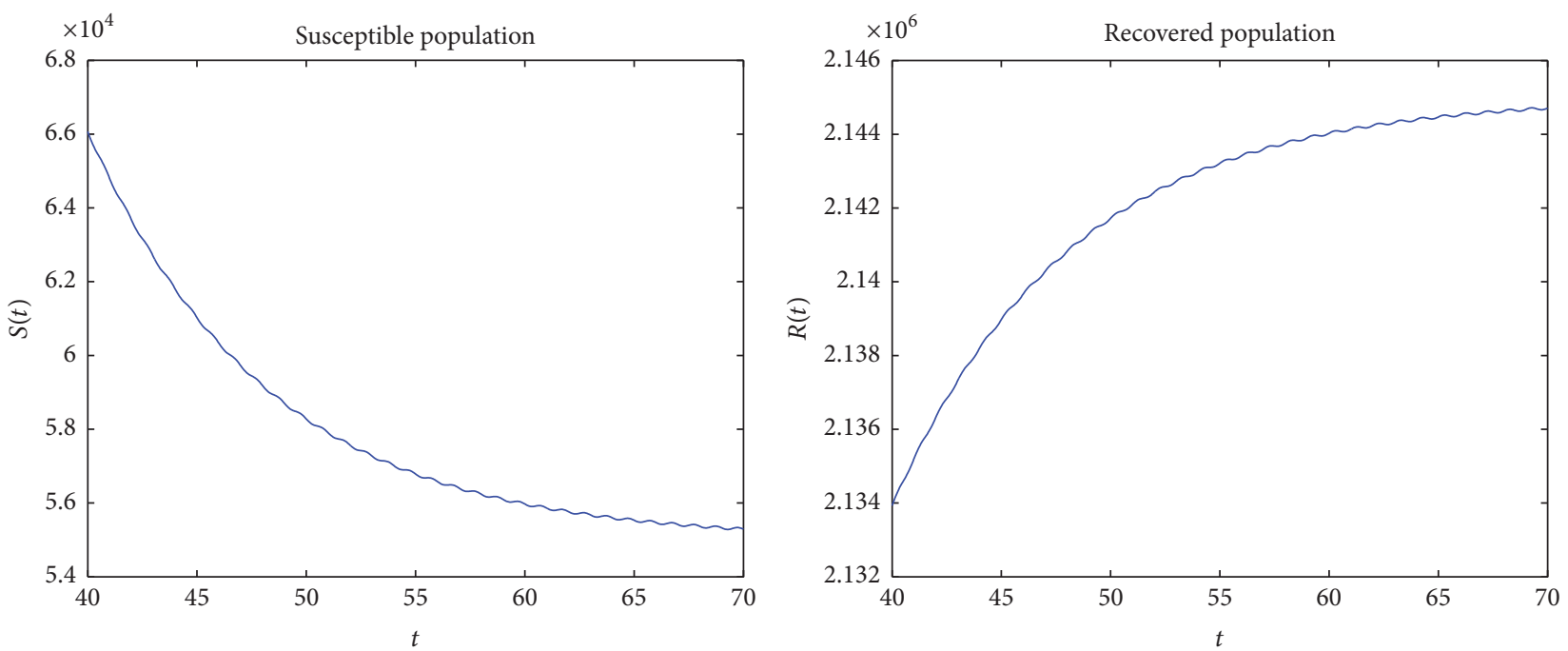

FIGURE 3: Solution of susceptible and recovered populations of SEIRS system when $\mathscr{R}_{0}<1$. We can see that $S$ approaches $\widehat{S}(t)$ (see Figure 1 ), and $R$ approaches $\widehat{R}(t)=N-\widehat{S}(t)$. Time $t$ is given in years.

Example 2. Now, choose $\beta_{0}=0.005$. As we can see in Figures 4 and 5, the solutions of system (1) remain persistent when $t$ tends to infinity; this fact suggests that $\mathscr{R}_{0}>1$ from Theorem 8. In fact, if we compute the basic reproduction number and its average (using the process described in example 1), $\mathscr{R}_{0}^{T}=3.298021580$ and $\mathscr{R}_{0} \in(2.7456,2.7457)$; therefore it is bigger than one. In fact, this shows the results of persistence obtained in Theorem 8 .

\section{Conclusion}

In this paper we presented a model with seasonal fluctuation with a general incidence function $S f(I)$ that includes the bilinear case $\beta S I$ (studied by [5]) and a family of saturated incidence rate of the form $\beta S I /\left(1+k I^{q}\right)$. We proved the existence of a disease-free periodic solution $(\widehat{S}(t), 0,0)$ and defined the basic reproduction number $\mathscr{R}_{0}$, proving that it is a threshold parameter for disease, in the sense that when $\mathscr{R}_{0}<$ 1 , the disease-free periodic solution is globally asymptotically stable, and when $\mathscr{R}_{0}>1$, the disease is persistent. A next step of this work is to consider a family of incidence rates more generally, changing $S f(I)$ by $f(S, I)$ and trying to obtain results of persistence and stability similar to the ones obtained in this work. Another interesting topic is to ask what the behavior of system at $\mathscr{R}_{0}=1$ is, in order to complete the analysis that we have made.

Several authors (e.g., $[10,11])$ define $\mathscr{R}_{0}$ as an average, which we denoted as $\mathscr{R}_{0}^{T}$ to distinguish between it and the basic reproduction number defined by [13], via the monodromy matrix (which is a real threshold parameter 

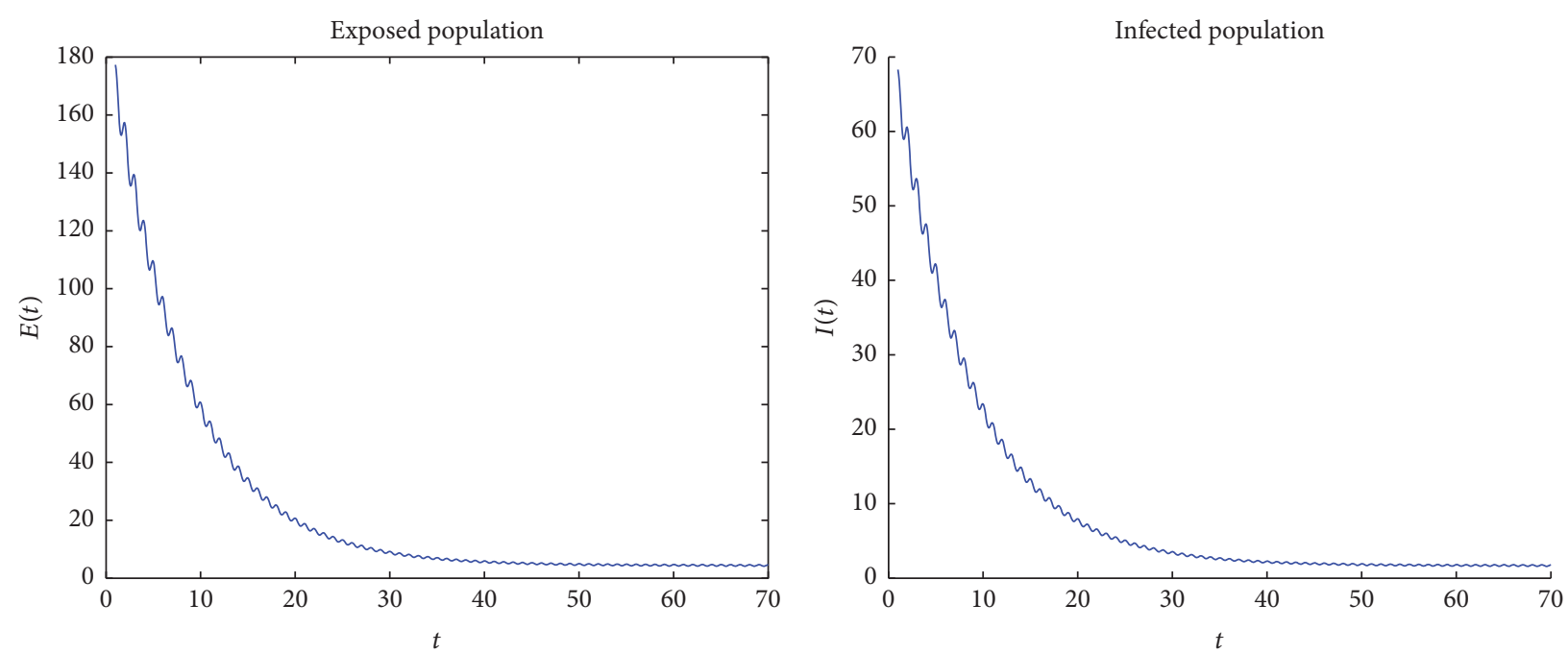

FIgURE 4: Solution of exposed and infected individuals of SEIRS system when $\mathscr{R}_{0}>1$. Both $E$ and $I$ remain persistent when time goes to infinity. Time $t$ is given in years.
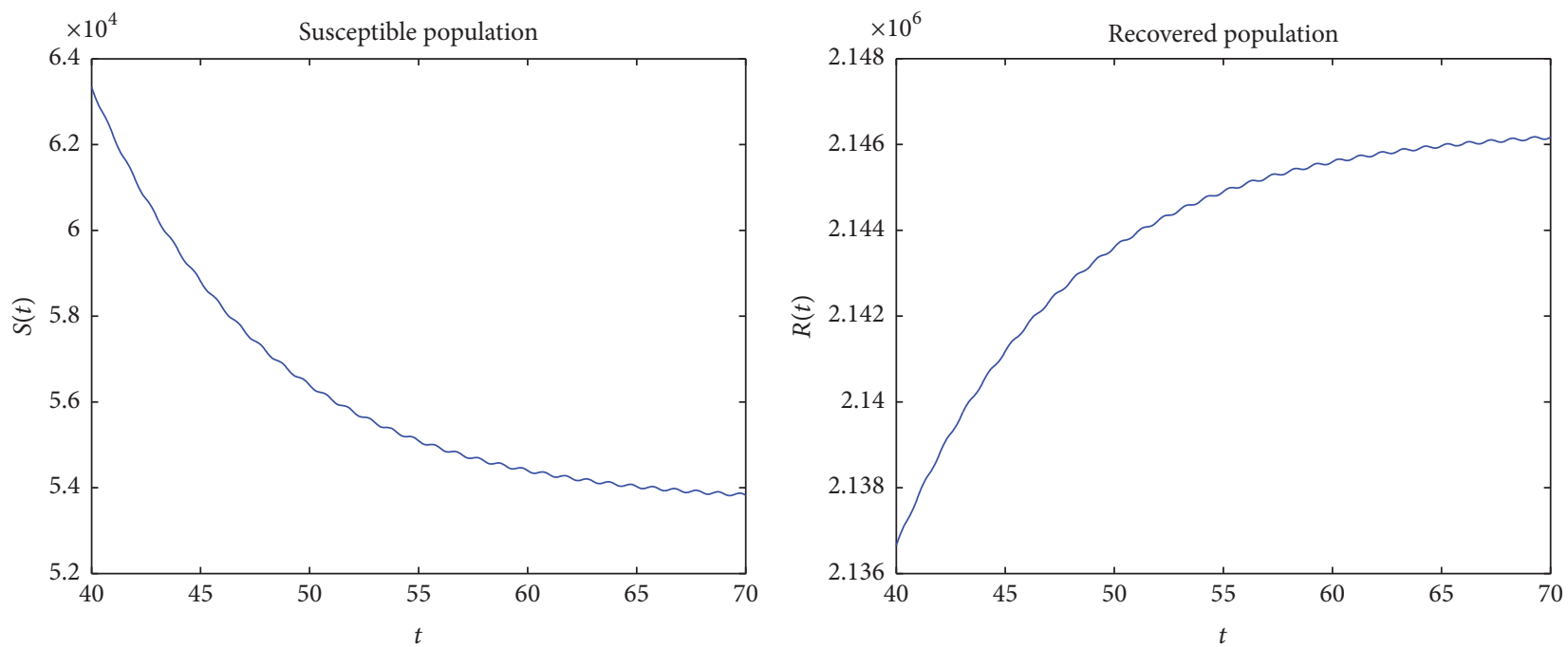

FIGURE 5: Solution of susceptible and recovered populations of SEIRS system when $\mathscr{R}_{0}>1$. Time $t$ is given in years.

for extinction and persistence of disease). We compute $\mathscr{R}_{0}^{T}$, approximate $\mathscr{R}_{0}$ (with the help of Taylor theorem), and compare these values, obtaining that $\mathscr{R}_{0}^{T}$ is not equal to $\mathscr{R}_{0}$; moreover $\mathscr{R}_{0}^{T}>\mathscr{R}_{0}$ in both examples (similar comparisons can be observed also in the works made by $[13,17])$. This fact suggests that the use of $\mathscr{R}_{0}^{T}$ for persistence overestimates the threshold. To emphasize this conclusion, it would be helpful to find an example where $\mathscr{R}_{0}<1$ but $\mathscr{R}_{0}^{T}>1$ and then compute the solutions to observe the behavior (we affirm that the disease will go extinct due to Theorem 6).

To obtain the estimation of $\mathscr{R}_{0}$ we used a code in Maple, which is based on numerical computing of $\rho_{1}=$ $\rho\left(W\left(L T, 0, \lambda_{i}\right)\right)$ until $\rho_{1} \sim 1$, where $\lambda_{i}=\lambda_{0}+\Delta_{\lambda} i, \Delta_{\lambda}$ is the step size, and the initial estimation $\lambda_{0}$ is taken as $R_{0}^{T}-\epsilon$. For this approximation we have used a Taylor expansion of the periodic solution $\widehat{S}(t)$; another interesting possibility could be varying the approximation used for $\mathscr{R}_{0}$, for example, changing the Taylor approach of $\widehat{S}(t)$ around $t=1$ instead of $t=0$. The graphs of the solutions were obtained with ODE 45 from Matlab, but other methods can be used to improve them, for example, Adomian methods or homotopy methods $[24,25]$. The Maple code used to estimate $\mathscr{R}_{0}$ is available for anyone who wants to use it.

\section{Appendix}

\section{A. Assumption on $\eta$ Used in Theorem 8}

Note that $\widehat{S}(t)$ has a positive minimum value $\min (\widehat{S}(t))$ (it is periodic, positive, and continuous, so it is bounded for $t \epsilon$ $[0, L T]$ and then for all $t>0)$ and we can choose a $\eta>0$ 
with the property $\min (\widehat{S}(t))>\eta$, sufficiently small such that $\widehat{S}(t)-\eta>0$.

\section{B. Periodic Solution of (61)}

For each $\alpha,(61)$ used in the proof of Theorem 8 is

$$
\begin{aligned}
\frac{d \bar{S}}{d t}= & N(\mu(1-p)+\delta)-2 \delta \alpha \\
& -\left(\beta(t) f^{\prime}(0) \alpha+\mu+r(t)+\delta\right) \widehat{S} .
\end{aligned}
$$

Solving the equation above, we arrive at the general solution

$$
\begin{aligned}
\bar{S}(t) & =e^{-\int_{t_{0}}^{t} p(s) d s}\left[\bar{S}\left(t_{0}\right)\right. \\
& \left.+(N(\mu(1-p)+\delta)-2 \delta \alpha) \int_{t_{0}}^{t} e^{\int_{t_{0}}^{s}(p(\zeta) d \zeta)} d s\right],
\end{aligned}
$$

where $p(s)=\beta(s) f^{\prime}(0) \alpha+\mu+r(s)+\delta$. We shall examine the behavior of an arbitrary solution $\bar{S}$. For each $n=0,1, \ldots$, we can use an initial time $\bar{t}_{0}=t_{0}+n L T$ with initial point $\bar{S}\left(\bar{t}_{0}\right)$ and see that

$$
\begin{gathered}
\bar{S}\left(t_{0}+(n+1) L T\right)=e^{-\int_{t_{0}+n L T}^{\left(t_{0}+n L T\right)+L T} p(s) d s}\left[\bar{S}\left(t_{0}+n L T\right)\right. \\
+(N(\mu(1-p)+\delta)-2 \delta \alpha) \\
\left.\cdot \int_{\left(t_{0}+n L T\right)}^{\left(t_{0}+n L T\right)+L T} e^{\int_{t_{0}+n L T}^{s}(p(\zeta) d \zeta) d s}\right] .
\end{gathered}
$$

Since $p(s)$ is a periodic function,

$$
\begin{aligned}
\int_{t_{0}+n L T}^{\left(t_{0}+n L T\right)+L T} p(s) d s & =\int_{t_{0}}^{t_{0}+L T} p(s) d s=\int_{0}^{L T} p(s) d s, \\
\int_{t_{0}+n L T}^{s} p(\zeta) d \zeta & =\int_{t_{0}}^{s-n L T} p(\zeta) d \zeta,
\end{aligned}
$$

where $s-n L T \geq t_{0}$. Then

$$
\begin{gathered}
\bar{S}\left(t_{0}+(n+1) L T\right)=e^{-\int_{t_{0}}^{\left(t_{0}+L T\right)} p(s) d s}\left[\bar{S}\left(t_{0}+n L T\right)\right. \\
+(N(\mu(1-p)+\delta)-2 \delta \alpha) \\
\left.\cdot \int_{\left(t_{0}+n L T\right)}^{\left(t_{0}+n L T\right)+L T} e^{\int_{t_{0}}^{s-L T}(p(\zeta) d \zeta)} d s\right] .
\end{gathered}
$$

And using the change of variable $u=s-L T$, we have

$$
\begin{aligned}
& \bar{S}\left(t_{0}+(n+1) L T\right)=e^{-\int_{t_{0}}^{\left(t_{0}+L T\right)} p(s) d s}\left[\bar{S}\left(t_{0}+n L T\right)\right. \\
& \left.\quad+(N(\mu(1-p)+\delta)-2 \delta \alpha) \int_{t_{0}}^{t_{0}+L T} e^{\int_{t_{0}}^{u}(p(\zeta) d \zeta)} d u\right] .
\end{aligned}
$$

Equation (B.6) gives a recursive relationship between the solution at $t_{0}+n L T$ and after $L T$ times. If we set $S_{n}=\bar{S}\left(t_{0}+\right.$ $n L T)$, then for each solution $\bar{S}$ this relationship is described by

$$
S_{n+1}=F\left(S_{n}\right),
$$

with $F$ being on the right side of (B.6). If we take $S_{i}$ and $S_{j}$, two different values of $S_{n}$, then

$$
\begin{aligned}
\left|F\left(S_{i}\right)-F\left(S_{j}\right)\right| & =e^{-\int_{T_{0}}^{t_{0}+L T} p(s) d s}\left|S_{i}-S_{j}\right| \leq\left|S_{i}-S_{j}\right| \\
& \leq e^{-(\mu+\delta) L T}\left|S_{i}-S_{j}\right| .
\end{aligned}
$$

Then, $F(S)$ is a contracting map, and by Banach fixed point theorem $F$ has a unique fixed point $S_{i}$ such that $S_{i+1}=$ $F\left(S_{i}\right)=S_{i}$ or, equivalently, $\bar{S}\left(t_{0}+i L T\right)=\bar{S}\left(t_{0}+(i+1) L T\right)$. This fixed point can be found for any $S$ that is a solution of a differential equation with arbitrary initial condition $S\left(t_{0}\right)$ at any time $t_{0}$. The fixed point has the form

$$
\begin{aligned}
& \bar{S}\left(t_{0}^{*}\right) \\
& =\frac{(N(\mu(1-p)+\delta)-2 \delta \alpha) \int_{t_{0}^{*}}^{t_{0}^{*}+L T}\left(e^{\int_{t_{0}}^{u} p(s) d s}\right) d u}{e^{\int_{0}^{L T} p(s) d s}-1} .
\end{aligned}
$$

Thus, define the function

$$
\begin{aligned}
& \widehat{S}(t) \\
& =\frac{(N(\mu(1-p)+\delta)-2 \delta \alpha) \int_{t}^{t+L T}\left(e^{\int_{t}^{u} p(s) d s}\right) d u}{e^{\int_{0}^{L T} p(s) d s}-1} .
\end{aligned}
$$

$\widehat{S}$ is a periodic function with period $L T$ and is continuously differentiable with respect to $t$. One can check (by computing the derivative) that $\widehat{S}(t)$ is a solution of differential equation, so by existence and uniqueness of solutions it can be rewritten as

$$
\begin{aligned}
\widehat{S}(t) & =e^{-\int_{0}^{t} p(s) d s}[\widehat{S}(0) \\
& \left.+(N(\mu(1-p)+\delta)-2 \delta \alpha) \int_{0}^{t} e^{\int_{0}^{s}(p(\zeta) d \zeta)} d s\right],
\end{aligned}
$$

with initial condition

$$
\widehat{S}(0)=\frac{(N(\mu(1-p)+\delta)-2 \delta \alpha) \int_{0}^{L T} e^{\int_{0}^{s}(p(\zeta) d \zeta)} d s}{e^{\int_{0}^{L T} p(s) d s}-1} .
$$

If we suppose the existence of another periodic solution $\widehat{S}_{2}(t)$, then using (B.6) we arrive at $\widehat{S}_{2}(0)=\widehat{S}(0)$, by uniqueness of solutions $\widehat{S}=\widehat{S}_{2}$, and the periodic solution is unique. Computing the difference $\bar{S}(t)-\widehat{S}(t)$, we have

$$
\bar{S}(t)-\widehat{S}(t)=e^{-\int_{0}^{t} p(s) d s}[\bar{S}(0)-\widehat{S}(0)],
$$

so, $\lim (\bar{S}(t)-\widehat{S}(t))=0$. Therefore, every solution $\bar{S}(t)$ converges to $\widehat{S}(t)$. 


\section{Assumption on $P^{m}$ Used in Theorem 8}

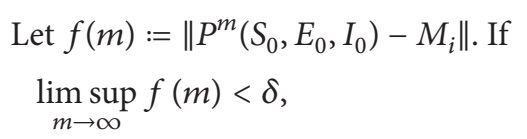

$$
\text { for some }\left(S_{0}, E_{0}, I_{0}\right) \in X_{0}, i=1,2 \text {, }
$$

then we have $L=\lim _{m \rightarrow \infty}\left(\sup _{n \geq m} f(n)\right)<\delta$. For all $\epsilon>0$ there exists a $M_{\epsilon}>0$ such that if $m \geq M_{\epsilon}$, then $-\epsilon<$ $\sup _{n \geq m} f(n)-L<\epsilon$. In particular, for $\epsilon=(\delta-L) / 2>0$ we have

$$
\sup _{n \geq m} f(n)-L<\delta-L
$$

or, equivalently, $\sup _{n \geq m} f(n)<\delta$ for $m \geq M_{\delta-L}$. Moreover, for all $n \geq m$ with $m \geq M_{\delta-L}$, we have $f(n)<\sup _{n \geq m} f(n)<\delta$. Therefore, $\left\|P^{n}\left(S_{0}, E_{0}, I_{0}\right)-M_{i}\right\|<\delta, \forall n \geq M_{\delta_{L}}$.

We can take $\left(S_{0}^{1}, E_{0}^{1}, I_{0}^{1}\right)=P^{M_{\delta-L}}\left(S_{0}, E_{0}, I_{0}\right)$ as initial condition and, therefore,

$$
\left\|P^{n}\left(S_{0}^{1}, E_{0}^{1}, I_{0}^{1}\right)-M_{i}\right\|<\delta, \quad \forall n \geq 0,
$$

making our assumption valid.

So, we can assume without loss of generality that $\left\|P^{m}\left(S_{0}, E_{0}, I_{0}\right)-M_{i}\right\|<\delta$ for all $m \geq 0$.

\section{Expression (71)}

From system (7) $d E / d t=\beta(t) S f(I)-(\mu+\sigma) E$, with $S(t)>$ $\widehat{S}(t)-\eta$ for $t>T$, so

$$
\frac{d E}{d t} \geq \beta(t)(\widehat{S}(t)-\eta) f(I)-(\mu+\sigma) E, \quad \text { for } t>T .
$$

Using assumption (A5) for $f(I)$ and positivity of $\widehat{S}(t)-\eta$, we have also

$$
\begin{aligned}
f(I) & (\widehat{S}(t)-\eta) \\
& \geq(\widehat{S}(t)-\eta)\left[I f^{\prime}(0)+\frac{1}{2} I^{2} f^{\prime \prime}(0)\right] .
\end{aligned}
$$

Therefore,

$$
\begin{aligned}
\frac{d E}{d t} \geq & \beta(t)(\widehat{S}(t)-\eta)\left[I f^{\prime}(0)+\frac{1}{2} I^{2} f^{\prime \prime}(0)\right] \\
& -(\mu+\sigma) E, \\
= & \beta(t)(\widehat{S}(t)-\eta) I f^{\prime}(0) \\
& +\frac{1}{2} \beta(t)(\widehat{S}(t)-\eta) f^{\prime \prime}(0) I^{2}-(\mu+\sigma) E .
\end{aligned}
$$

$0<I<\alpha$ and $f^{\prime \prime}(0) \leq 0$, so $I^{2}<\alpha I$ and $f^{\prime \prime}(0) I^{2} \geq f^{\prime \prime}(0) \alpha I$; applying this we arrive at

$$
\begin{aligned}
\frac{d E}{d t} \geq & \beta(t)(\widehat{S}(t)-\eta) I f^{\prime}(0) \\
& +\frac{1}{2} \beta(t)(\widehat{S}(t)-\eta) f^{\prime \prime}(0) \alpha I, \\
\frac{d I}{d t}= & \sigma E-(\mu+\sigma) I .
\end{aligned}
$$

This expression can be written as (71).

\section{Conflicts of Interest}

The authors declare that they have no conflicts of interest.

\section{Acknowledgments}

This article was supported in part by Mexican SNI under Grants nos. 15284 and 33365.

\section{References}

[1] W. O. Kermack and A. G. McKendrick, "A contribution to the mathematical theory of epidemics," Proceedings of the Royal Society A Mathematical, Physical and Engineering Sciences, vol. 115 , no. 772, pp. 700-721, 1927.

[2] L. Wang, X. Zhang, and Z. Liu, "An SEIR Epidemic Model with Relapse and General Nonlinear Incidence Rate with Application to Media Impact," Qualitative Theory of Dynamical Systems, pp. $1-21$.

[3] V. Capasso and G. Serio, "A generalization of the KermackMcKENdrick deterministic epidemic model," Mathematical Biosciences, vol. 42, no. 1-2, pp. 43-61, 1978.

[4] Z. Bai, "Threshold dynamics of a periodic SIR model with delay in an infected compartment," Mathematical Biosciences and Engineering, vol. 12, no. 3, pp. 555-564, 2015.

[5] Z. Bai and Y. Zhou, "Global dynamics of an SEIRS epidemic model with periodic vaccination and seasonal contact rate," Nonlinear Analysis: Real World Applications, vol. 13, no. 3, pp. 1060-1068, 2012.

[6] A. Kaddar, S. Elkhaiar, and F. Eladnani, "Global Asymptotic Stability of a Generalized SEIRS Epidemic Model," Differential Equations and Dynamical Systems, pp. 1-11.

[7] M. A. Khan, Y. Khan, Q. Badshah, and S. Islam, "Global stability of SEIVR epidemic model with generalized incidence and preventive vaccination," International Journal of Biomathematics, vol. 8, no. 6, Article ID 1550082, 2015.

[8] M. A. Khan, Y. Khan, T. W. Khan, and S. Islam, "Dynamical system of a SEIQV epidemic model with nonlinear generalized incidence rate arising in biology," International Journal of Biomathematics, vol. 10, no. 7, Article ID 1750096, 2017.

[9] P. van den Driessche and J. Watmough, "Reproduction numbers and sub-threshold endemic equilibria for compartmental models of disease transmission," Mathematical Biosciences, vol. 180, no. 1, pp. 29-48, 2002.

[10] L. Li, Y. Bai, and Z. Jin, "Periodic solutions of an epidemic model with saturated treatment," Nonlinear Dynamics, vol. 76, no. 2, pp. 1099-1108, 2014.

[11] Y. Xu and L. Li, "Global exponential stability of an epidemic model with saturated and periodic incidence rate," Mathematical Methods in the Applied Sciences, vol. 39, no. 13, pp. 36503658, 2016.

[12] N. Bacaër and S. Guernaoui, "The epidemic threshold of vector-borne diseases with seasonality. The case of cutaneous leishmaniasis in Chichaoua, Morocco," Journal of Mathematical Biology, vol. 53, no. 3, pp. 421-436, 2006.

[13] W. Wang and X.-Q. Zhao, "Threshold dynamics for compartmental epidemic models in periodic environments," Journal of Dynamics and Differential Equations, vol. 20, no. 3, pp. 699-717, 2008. 
[14] I. A. Moneim and D. Greenhalgh, "Use of a periodic vaccination strategy to control the spread of epidemics with seasonally varying contact rate," Mathematical Biosciences and Engineering, vol. 2, no. 3, pp. 591-611, 2005.

[15] D. Posny and J. Wang, "Modelling cholera in periodic environments," Journal of Biological Dynamics, vol. 8, no. 1, pp. 1-19, 2014.

[16] N. Bacaër, "Approximation of the basic reproduction number R0 for vector-borne diseases with a periodic vector population," Bulletin of Mathematical Biology, vol. 69, no. 3, pp. 1067-1091, 2007.

[17] C. D. Mitchell, Reproductive Numbers for Periodic Epidemic Systems [PhD thesis], University of Texas, 2016.

[18] F. Zhang and X.-Q. Zhao, "A periodic epidemic model in a patchy environment," Journal of Mathematical Analysis and Applications, vol. 325, no. 1, pp. 496-516, 2007.

[19] H. L. Smith and P. Waltman, The Theory of the Chemostat, Cambridge University Press, 1995.

[20] T. Kato, Perturbation Theory for Linear Operators, vol. 132 of Grundlehren der Mathematischen Wissenschaften, Springer, New York, NY, USA, 1966.

[21] X. Q. Zhao, Dynamical Systems in Population Biology, Springer Science \& Business Media, New York, NY, USA, 2003.

[22] Y. Yang, S. Ruan, and D. Xiao, "Global stability of an agestructured virus dynamics model with Beddington-DeAngelis infection function," Mathematical Biosciences and Engineering, vol. 12, no. 4, pp. 859-877, 2015.

[23] Y. Nakata and T. Kuniya, "Global dynamics of a class of SEIRS epidemic models in a periodic environment," Journal of Mathematical Analysis and Applications, vol. 363, no. 1, pp. 230237, 2010.

[24] Y. Khan and M. Fardi, "A new efficient multi-parametric homotopy approach for two-dimensional Fredholm integral equations of the second kind," Hacettepe Journal of Mathematics and Statistics, vol. 44, no. 1, pp. 93-99, 2015.

[25] Y. Khan, K. Sayevand, M. Fardi, and M. Ghasemi, "A novel computing multi-parametric homotopy approach for system of linear and nonlinear Fredholm integral equations," Applied Mathematics and Computation, vol. 249, pp. 229-236, 2014. 


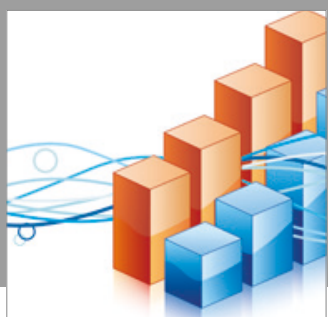

Advances in

Operations Research

vatersals

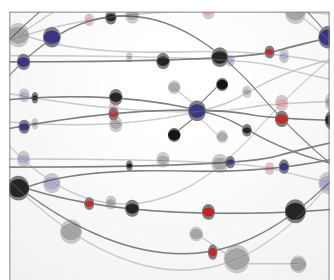

\section{The Scientific} World Journal
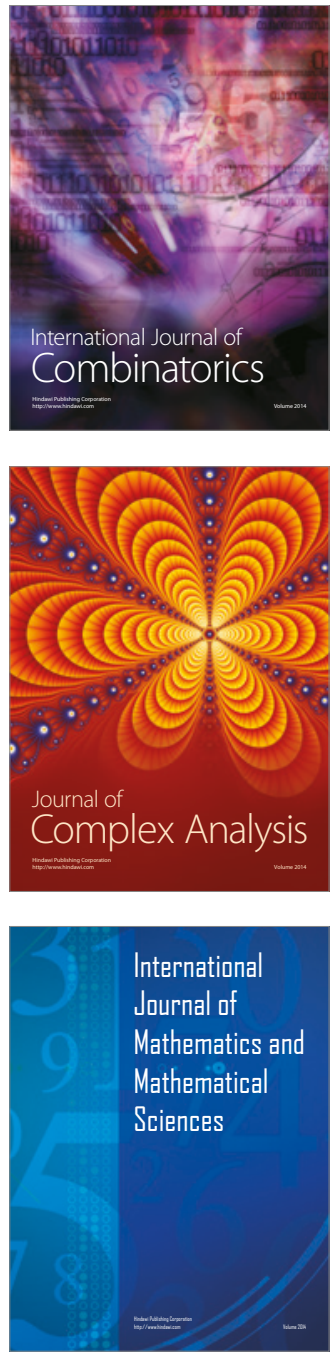
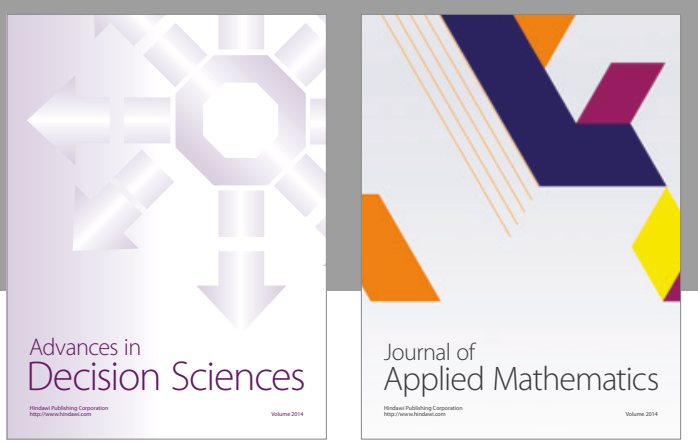

Algebra

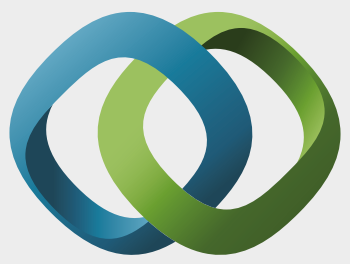

\section{Hindawi}

Submit your manuscripts at

https://www.hindawi.com
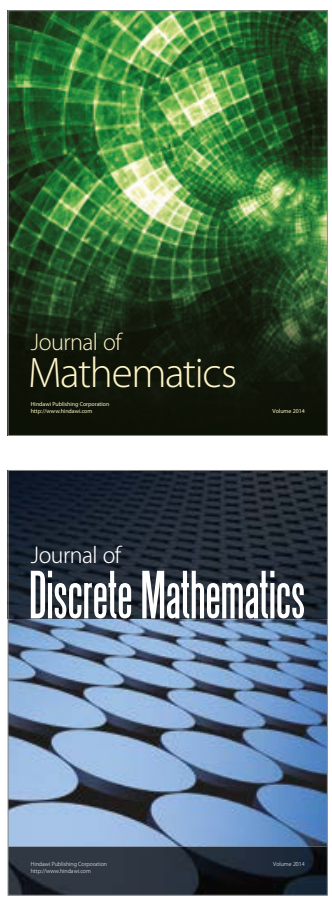

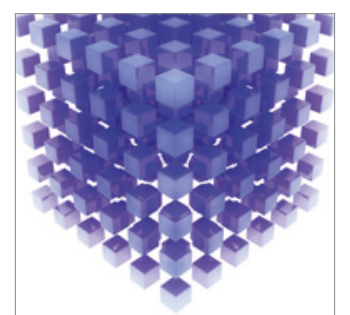

Mathematical Problems in Engineering
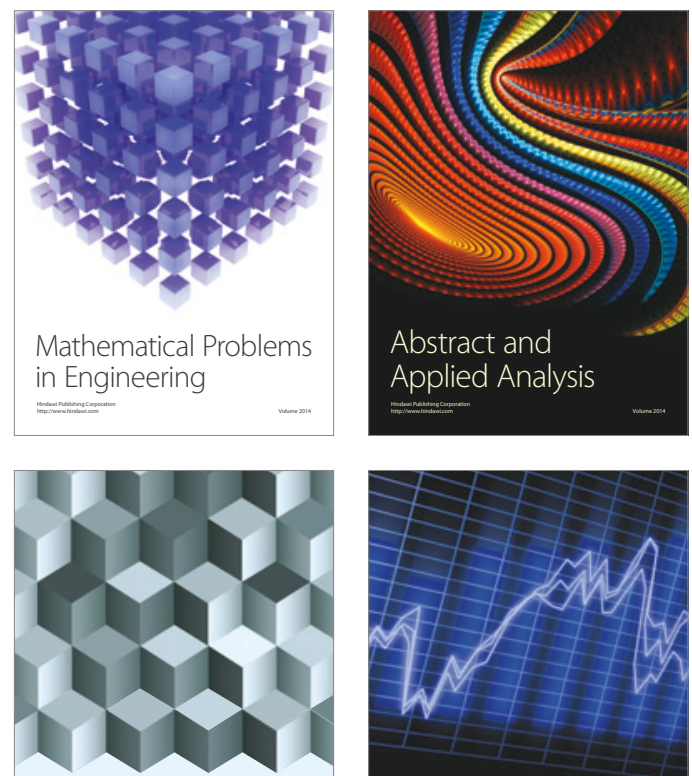

Journal of

Function Spaces

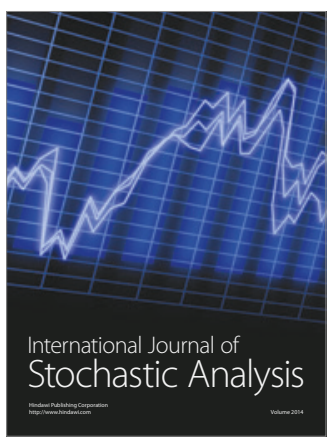

Probability and Statistics
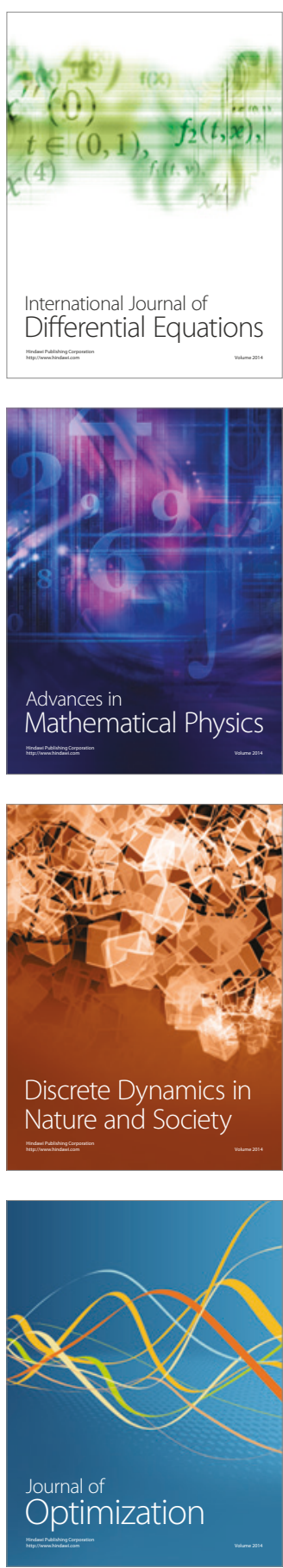